\title{
(HIV-)Prävention beginnt in der Arztpraxis
}

Der Prävention wird heute in vielen Bereichen eine grosse Bedeutung beigemessen. Prävention von Gesundheitsproblemen setzt die Kenntnis der auslösenden Faktoren einer Krankheit voraus. So waren es die Mediziner, die als erste auf die Probleme des Tabakrauchens aufmerksam machten. Auch die Folgen des Übergewichts und der Bewegungsarmut werden in der Arztpraxis thematisiert. Wir Ärztinnen und Ärzte verfügen über eine enorme Erfahrung: Wir sehen so viele Einzelschicksale wie kaum eine andere Berufsgruppe. Persönliche Betroffenheit motiviert uns zur Präventionsarbeit in unserem Arbeitsgebiet: Die Augenärztin avisiert die Früherkennung des Glaukoms, der Pneumologe engagiert sich in der Tabakprävention usw. Für Internisten und Allgemeinpraktiker sind die meisten Präventionsfelder ein tägliches Thema. In zahlreichen Praxen sitzen präventiv aktive Ärztinnen und Ärzte, die mit grossem Engagement und ohne grosses Aufheben einen wichtigen Anteil der Präventionsarbeit leisten.

Mitte der 80er Jahre, als die Aidsepidemie in der Schweiz ihren Anfang nahm, waren es die HIV-Spezialisten der ersten Stunde, die sich engagiert mit Vorträgen, Medienberichten und in konzeptionellen Arbeiten für die HIV-Prävention einsetzten. Von Anfang an haben sich diese Ärztinnen und Ärzte auch für die Verbindung von HIV-Test und Testberatung eingesetzt. Sie taten dies und tun dies auch heute noch motiviert durch ihre persönliche Erfahrung mit HIV-/aidsbetroffenen Menschen. Aus Erfahrung wissen wir, dass noch allzuoft mangelndes Wissen und Fehlverhalten zu einer HIV-Infektion führen. Ebenfalls häufig beobachten wir Fehlinterpretationen von negativen HIV-Testresultaten.

Daten der Schweizerischen Gesundheitsbefragung und des Bundesamtes für Gesundheit zeigen, dass in der Schweiz jährlich 300000 HIV-
Testungen ausserhalb des Blutspendewesens veranlasst werden, ein Grossteil davon in Arztpraxen. Die HIV-Testung eignet sich hervorragend für ein kurzes Präventionsgespräch. Selbst wenn dieses Gespräch nur jedes 3. Mal 3 Minuten dauert, sind dies 5000 Stunden, die jährlich in Beratungsgespräche investiert werden. Der damit verbundene Aufwand ergäbe einen finanziell grösseren Betrag, als dem BAG für seine ganze HIV-Präventionsarbeit zur Verfügung steht.

Wir Ärztinnen und Ärzte sind uns der Bedeutung dieser Präventionsarbeit bewusst und setzen uns daher auch dafür ein, dass diese Arbeit professionell gemacht wird. Im BAG-Bulletin vom Juli wird eine Arbeit publiziert, welche die HIV-Testberatung in der Arztpraxis untersuchte. Die Resultate sind erfreulich und anregend zugleich. Sie zeigen, dass sich viele Ärzte und Ärztinnen ihrer enormen Bedeutung für (HIV-)Präventionsaufgaben bewusst sind. Sie zeigen aber auch, dass die Testberatung vielerorts noch optimiert werden kann.

Als Beitrag zur Optimierung der Präventionsberatung hat die Fachkommission Klinik und Therapie HIV/Aids (FKT) ein kurzes Vademecum verfasst, das ebenfalls im BAG-Bulletin und hier in einer Kurzversion publiziert wird. Im Sinne einer Anregung soll der Text Ideen für das Beratungsgespräch liefern. Zudem sind die zentralen Botschaften zur HIV-Prävention zusammengefasst, damit diese weiterhin so konsistent wie möglich kommuniziert werden. Konsultieren Sie den Text, wenn Sie das nächste Mal einen HIV-Test veranlassen, und helfen Sie mit, dass das Anliegen Prävention in der Arztpraxis weiterhin einen hohen Stellenwert bewahrt!

Pietro Vernazza, Präsident Fachkommission Klinik und Therapie HIV/Aids (FKT), St. Gallen 